\title{
Características dos Serviços de Computação em Nuvem Usados por Organizações Brasileiras
}

\author{
Neilson Carlos Leite Ramalho, Edmir Parada Vasques Prado \\ ${ }^{1}$ Escola de Artes, Ciências e Humanidades - Universidade de São Paulo (USP) \\ neilson@usp.br, eprado@usp.br
}

\begin{abstract}
Cloud Computing (CC) is one of the most discussed topics among IT professionals today. With a strong economic appeal, CC makes possible the idea of computing as a utility, in which computing resources (processing power and storage, for example) can be consumed and paid with the same convenience as electricity. This research analyzes CC adoption by Brazilian companies of all sizes and sectors that have adopted at least one IT service in CC model. This paper used a not randomly picked sample with 96 cases. This research identified the major cloud computing services used in Brazil as well as their characteristics, motivations for contracting the service provider and the impact of those services in the organization.
\end{abstract}

Resumo. A Computação em Nuvem (CN) é um dos temas mais abordados por profissionais de TI atualmente. Com um forte apelo econômico, a CN torna possível a ideia da computação como uma utilidade, onde recursos computacionais (processamento e armazenamento, por exemplo) podem ser consumidos e pagos com a mesma conveniência que a energia elétrica. Esta pesquisa analisa a adoção da $C N$ no Brasil por empresas privadas de diversos portes e setores que tenham adotado pelo menos um serviço de TI no modelo de CN. A pesquisa utilizou uma amostra não probabilística contendo 96 casos. Foram identificados os principais serviços de CN utilizados no Brasil, suas características, as motivações para contratação do fornecedor e o impacto organizacional de tais serviços.

\section{Introdução}

A Computação em Nuvem $(\mathrm{CN})$ constitui, atualmente, um dos temas mais abordados na área da Tecnologia da Informação (TI). Diversos grupos de pesquisa ao redor do mundo têm concentrado seus esforços visando entender este novo conceito e suas implicações para as organizações e para a sociedade. Com um forte apelo econômico, a $\mathrm{CN}$ torna possível a ideia da computação como uma utilidade, na qual recursos computacionais podem ser consumidos e pagos com a mesma conveniência que a energia elétrica. A CN é então a concretização da computação como utilidade.

Por oferecer às empresas a oportunidade de dissociar suas necessidades de TI de sua infraestrutura, a $\mathrm{CN}$ é capaz de oferecer, em longo prazo, economia às empresas, incluindo a redução de custos de infraestrutura e modelos de pagamento baseados em utilização de serviços (pagamento somente pelo que é usado). Adotando a $\mathrm{CN}$, as empresas podem tirar proveito do uso de serviços em um modelo sob demanda. A 
redução de despesas com capital inicial é importante, o que permite às empresas maior flexibilidade com novos serviços de TI (ISACA, 2009).

Dada a importância da $\mathrm{CN}$ no mundo e sua relevância para o Brasil como um país em desenvolvimento, é pertinente identificar como esse novo paradigma têm sido explorado e utilizado no cenário nacional. Como consequência, o objetivo geral deste trabalho é descrever as características dos serviços de $\mathrm{CN}$ usados por organizações brasileiras. Dentre as perguntas a serem respondidas estão: (1) quais serviços de CN são oferecidos no mercado e quais as suas características? (2) quais as motivações das organizações na escolha do fornecedor de CN? e (3) qual a importância para a organização dos serviços de $\mathrm{CN}$ contratados?

\section{Computação em Nuvem}

Esta seção apresenta os conceitos gerais da $\mathrm{CN}$, as classificações quanto aos tipos de serviços oferecidos e as formas de disponibilização. Além disso, apresenta um quadro de referência para analisar o impacto operacional e estratégico da $\mathrm{CN}$.

\subsection{Definição e Caracterização}

Existem diversas definições de $\mathrm{CN}$ na literatura, algumas convergentes para um conceito comum e outras com discrepâncias que, em muitos casos, são frutos de estratégias de Marketing. Armbrust et al. (2009), por exemplo, definem CN como a união entre as aplicações disponibilizadas como serviços na Internet, o hardware e sistemas de software nos Centro de Processamento de Dados (CPD) usados para prover tais serviços. Entretanto, Jeffery e Neidecker-Lutz (2010) abordam o conceito de CN sob outro ponto de vista, e definem a CN como um ambiente de execução que envolve múltiplas partes interessadas e fornece recursos com diferentes granularidades, de maneira elástica, medida e com um nível especificado de qualidade de serviço.

Vaquero et al. (2009) estudaram mais de vinte definições do termo CN para chegar a um consenso sobre a definição, e afirmam que o conceito ainda está sofrendo modificações. A definição de $\mathrm{CN}$ adotada neste trabalho tem como base a definição proposta por Mell e Grance (2011, p.1): “a CN é um modelo para permitir acesso de rede ubíquo, conveniente, e sob demanda a um repositório compartilhado de recursos computacionais, aparentemente infinitos e podem ser rapidamente provisionados e liberados com esforço mínimo de gerenciamento ou interação com o provedor de serviços."

\subsection{Classificação dos Serviços de Computação em Nuvem (CN)}

A CN envolve um conjunto de serviço de naturezas distintas. Com isso, é importante organizar e classificar esses serviços tendo como base as suas características. Na literatura, os dois mecanismos de classificação mais usados referem-se aos tipos de serviços oferecidos e a maneira como a $\mathrm{CN}$ é disponibilizada ao usuário final.

a) Tipos de serviços oferecidos. Quanto aos tipos de serviços oferecidos em CN, Motahari-Nezhad, Stephenson e Singhal (2009) sugerem a seguinte classificação:

- Infraestrutura como Serviço, Infrastructure as a Service (IaaS) - São recursos de hardware que são oferecidos como serviços para usuários finais. 
- Plataforma como Serviço, Platform as a Service (PaaS). É o oferecimento, na Internet, da infraestrutura de apoio para o ciclo de desenvolvimento de uma aplicação, desde o levantamento dos casos de uso, definição da arquitetura, codificação, testes e operação até a manutenção.

- Software como Serviço, Software as a Service (SaaS)- Conforme descrito por Armbrust et al. (2009), neste modelo, as aplicações são oferecidas como serviços na Internet, quebrando o modelo tradicional de software sob encomenda.

- Banco de dados como Serviço, Database as a Service (DaaS) - É o oferecimento de banco de dados como serviços. Geralmente utiliza uma arquitetura propícia para atender a diversos usuários, onde os dados desses usuários são armazenados em uma única tabela física.

As quatro classificações supracitadas são as mais abordadas na literatura. Todavia, novas classificações têm surgido, conforme aumenta a variedade de serviços oferecidos via CN. Como isso, foi cunhando o termo $X$ as a Service, ou Everything as a Service, que ilustram o conceito de Tudo como Serviço, conforme descrito por Schaffer (2009).

b) Forma de disponibilização ao usuário final. Em relação à forma como a CN é disponibilizada ao usuário final, Armbrust et al. (2009) e Jeffery e Neidecker-Lutz (2010) defendem as seguintes categorias:

- Nuvens públicas. Os serviços são disponibilizados na Internet e o usuário paga somente pelo que usa. Desse modelo de negócio, surge o conceito de computação como utilidade.

- Nuvens privadas. São os datacenters internos das organizações que não são disponibilizados publicamente. Motahari-Nezhad, Stephenson e Singhal (2009) argumentam que a CN é o resultado de uma evolução natural da infraestrutura de TI das empresas.

- Nuvens híbridas. Constituem uma composição de duas ou mais infraestruturas de CN (pública, privada ou comunitária), sendo que as entidades ainda se comportam como únicas, porém ligadas por tecnologias proprietárias ou padronizadas que permitem a portabilidade de dados e de aplicação.

- Nuvens comunitárias. A infraestrutura de $\mathrm{CN}$ é disponibilizada para uso exclusivo de uma comunidade específica de consumidores em organizações com objetivos e preocupações em comum, tais como: missão, requisitos de segurança, políticas, dentre outras.

- Nuvens de propósitos específicos. As nuvens de propósitos específicos fornecem serviços direcionados a casos de uso específicos e funcionalidades dedicadas.

\subsection{CN e o Impacto Estratégico e Operacional na Organização}

Segundo Applegate et al. (2003), a importância da TI nas organizações pode ser caracterizada em duas dimensões: o impacto da TI nas operações e na estratégia da organização. Os autores então criaram um quadro que relaciona níveis de cada uma 
dessas dimensões com quatro categorias de firma. Segundo os autores, essas quatro categorias ajudam a moldar a abordagem que será usada para identificar oportunidades, definir e desenvolver estratégias de negócio por meio da TI, e organizar e gerenciar ativos e recursos humanos. A Figura 1 ilustra as quatro categorias de acordo com as dimensões sugeridas e as recomendações quanto à estratégica de terceirização. A CN pode ser entendida como um processo de terceirização e, portanto, as estratégias relacionadas a $\mathrm{CN}$ podem ser analisadas por esse quadro de referência.

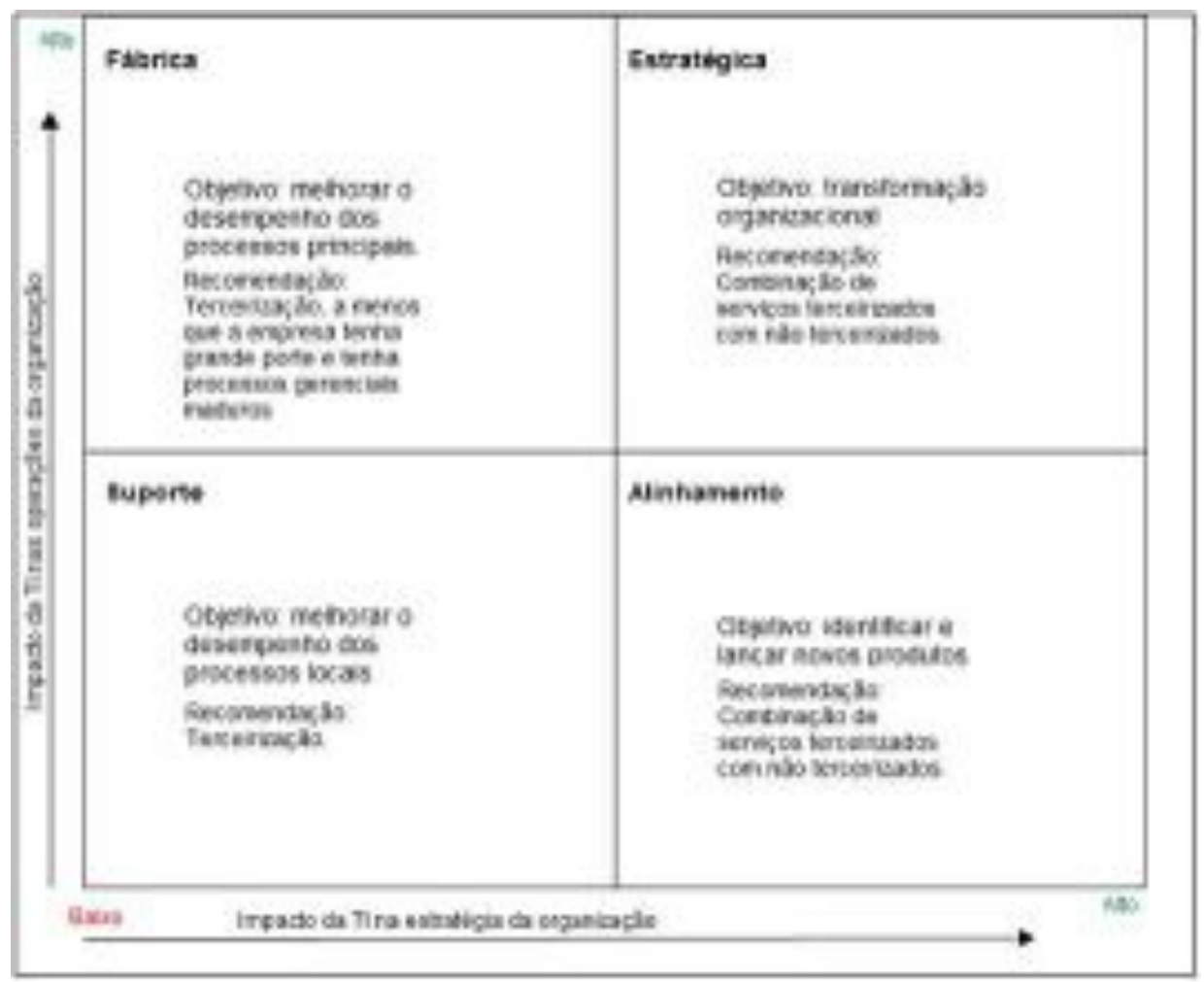

Figura 1. Impacto da TI nas organizações e as estratégias de terceirização

Fonte: adaptado de Applegate et al. (2003, p. 8)

O eixo vertical representa o impacto da TI nas operações, enquanto que no horizontal está representada a importância da TI para a estratégia da organização. Esses dois eixos contém quatro categorias recomendadas de terceirização:

- Suporte. Firmas nesta categoria são caracterizadas por terem baixa dependência operacional e estratégica de TI. Para este caso, recomenda-se a terceirização, especialmente para firmas de grande porte;

- Fábrica. O perfeito funcionamento das operações de TI é crucial para o desempenho das atividades organizacionais. Para firmas neste quadrante a recomendação é terceirizar, a menos que se trate de firmas de grande porte e com processos gerenciais maduros;

- Alinhamento. Nesta categoria, as iniciativas de negócio proporcionadas pela TI são críticas para o posicionamento estratégico da organização no futuro. As firmas deste quadrante devem combinar serviços terceirizados com não terceirizados. $\mathrm{Na} \mathrm{CN}$ a estratégia mais usual é terceirizar serviços não 
estratégicos, tais como serviços de hospedagem, e e-mail corporativo, por exemplo;

- Estratégica. Firmas neste quadrante também devem combinar serviços terceirizados com não terceirizados.

\section{Método da Pesquisa}

Nesta seção estão descritos as características e os procedimentos metodológicos adotados na pesquisa. Cabe resaltar que embora a $\mathrm{CN}$ esteja presente em empresas de diversos setores, este estudo ficou restrito às empresas privadas com pelo menos um serviço de TI no modelo de CN.

\subsection{Características da Pesquisa}

Esta pesquisa caracteriza-se por ser um estudo descritivo. Na pesquisa descritiva, um pesquisador inicia com um tema bem definido e conduz um estudo para descrevê-lo com acurácia, sendo que o resultado é um quadro detalhado do tema (Neuman, 2007). Além disso, na visão de Babbie (2007), os estudos descritivos raramente estão limitados a meros propósitos descritivos, e os pesquisadores geralmente examinam a existência dos padrões encontrados e suas implicações.

A estratégia de pesquisa adotada foi o survey (enquete). Isto porque, segundo Yin (2010), o survey é adequado para as situações onde: não se exige controle sobre eventos comportamentais; e o foco é em acontecimentos contemporâneos.

\subsection{Plano Amostral e Coleta de Dados}

A população pesquisada foi composta por funcionários ligados ao departamento de TI, sejam eles gestores ou analistas técnicos. Porém, foi exigido que o respondente estivesse familiarizado com as características básicas dos serviços de TI usados pela organização.

Os dados foram coletados usando o método de survey. Utilizou-se um questionário como instrumento de coleta de dados, que foi enviado por e-mail. Os resultados foram armazenados em uma planilha, e posteriormente importados para o software estatístico SPSS.

O questionário foi dividido em duas partes: características da organização e características dos serviços contratados. Os dados foram coletados no segundo semestre de 2012. O questionário englobou predominantemente questões fechadas com algumas questões em forma de campo de texto, como por exemplo o nome do serviço de $\mathrm{CN}$ utilizado, o tempo de experiência em TI e o nome da cidade do respondente.

Foi realizado um pré-teste com uma amostra de 20 questionários. Após a análise dessas respostas iniciais, foram feitos acertos no questionário para aos problemas de entendimento por parte dos respondentes.

\section{Análise de Dados}

Esta seção apresenta a análise dos dados coletados usando técnicas uni e multivariadas. A amostra é composta por 96 respondentes. Os dados contemplam resultados sobre o perfil do respondente, da organização e dos serviços de CN usados pelas organizações. 


\subsection{Características do respondente}

O questionário englobou perguntas de cunho técnico e gerencial. Com isso, tanto gestores quando analistas técnicos puderam responder a pesquisa. Duas questões estão relacionadas ao perfil do entrevistado: tempo de experiência na área de TI e nível hierárquico. No primeiro caso, a média foi de quase 15 anos de experiência, porém com alto desvio padrão (9 anos). Quanto ao segundo caso, 31 respostas foram de profissionais de nível técnico, 17 foram de coordenadores ou supervisores, nove foram de gerentes e 39 de diretores ou presidentes.

\subsection{Características das organizações}

As características das organizações pesquisadas são representadas por oito varáveis.

a) Localização. A localização está representada por duas variáveis: cidade (O1) e estado $(\mathrm{O} 2)$. A Tabela 1 apresenta as frequências absoluta, relativa e acumulada para cada região brasileira.

Tabela 1. Distribuição da amostra por região

\begin{tabular}{|c|r|r|r|}
\hline \multirow{2}{*}{ Região } & \multicolumn{3}{|c|}{ Frequências } \\
\cline { 2 - 4 } & Absoluta & Relativa (\%) & Acumulada (\%) \\
\hline Sudeste & 78 & 81,25 & 81,25 \\
\hline Sul & 8 & 8,33 & 89,58 \\
\hline Centro-Oeste & 6 & 6,25 & 95,83 \\
\hline Nordeste & 4 & 4,17 & 100,00 \\
\hline Norte & 0 & 0,00 & 100,00 \\
\hline
\end{tabular}

b) Porte e setor de atuação. Na amostra predominaram as empresas do setor de serviços com faturamento menor que $\mathrm{R} \$ 16$ milhões anuais $(54,3 \%$ dos resultados), Ainda no setor de serviços, a amostra apresentou 50,5\% de empresas cujo tempo de mercado é maior que 10 anos e 37,9\% de empresas de pequeno porte. Este resultado mostra que, embora a literatura relate que a $\mathrm{CN}$ traz benefícios especialmente para pequenas e médias empresas (que por meio da $\mathrm{CN}$ teriam acesso à infraestrutura serviços de TI sem altos investimentos antecipados), a realidade brasileira mostra ligeira predominância de pequenas empresas $(48,4 \%)$ sobre grandes empresas $(31,6 \%)$. Com base nos dados é possível verificar que há espaço para crescimento da $\mathrm{CN}$ entre as empresas de médio porte. A Tabela 2 resume as relações entre o setor de atuação e o faturamento, o tempo de mercado e o porte da organização.

Tabela 2. Faturamento, tempo de mercado e porte das organizações

\begin{tabular}{|c|c|c|c|c|c|c|c|c|c|c|c|}
\hline \multirow[t]{2}{*}{ Setor } & \multirow[t]{2}{*}{ Qtde } & \multicolumn{3}{|c|}{$\begin{array}{c}\text { Faturamento } \\
\text { (em R\$ milhões) }\end{array}$} & \multicolumn{3}{|c|}{$\begin{array}{c}\text { Tempo de Mercado } \\
\text { (em anos) } \\
\end{array}$} & \multicolumn{3}{|c|}{ Porte } & \multirow[t]{2}{*}{ Total } \\
\hline & & $<16$ & $16-90$ & $>90$ & 1 a 5 & 5 a 10 & $>10$ & $\mathbf{P}$ & $\mathbf{M}$ & $\mathbf{G}$ & \\
\hline Comércio & (9) & 8.7 & 0.0 & 0.0 & 1.1 & 0.0 & 8. & 5.3 & 1.1 & 3.2 & 9.5 \\
\hline Industria & (14) & 8.7 & 0.0 & 0.0 & 1.1 & 0.0 & 8. & 5.3 & 1.1 & 3.2 & 9.5 \\
\hline Serviço & (72) & 54.3 & 9.8 & 12.0 & 12.6 & 12.6 & 50.5 & 37.9 & 14.7 & 23.2 & 75.8 \\
\hline Total & (95) & 70.7 & 13.0 & 16.3 & 14.7 & 13.7 & 71.6 & 48.4 & 20.0 & 31.6 & 100.0 \\
\hline
\end{tabular}




\subsection{Características dos serviços de $\mathrm{CN}$}

Esta seção descreve as características dos serviços de $\mathrm{CN}$ utilizados pela organização.

a) Serviços utilizados sob a abordagem de $\mathbf{C N}$. Dos serviços utilizados houve destaque para aplicações mais tradicionais em TI, como e-mail, armazenamento, hospedagem e softwares de escritório no geral (planilhas, processadores de texto, apresentações). A Tabela 4 apresenta as aplicações que se destacaram. Abaixo segue um resumo delas:

- E-mail externo (na nuvem) com o domínio da empresa. Com $61.86 \%$, esta é a categoria de mais utilizada pelas organizações;

- Hospedagem de sites, portais e blogs. Com $58.76 \%$ de adoção, a hospedagem na nuvem é amplamente utilizada porque o próprio conceito de hospedagem já é algo consolidado em TI. Os principais fornecedores do mercado também disponibilizam ferramentas que tornam a utilização desse serviço muito semelhante à hospedagem tradicional;

- Ferramentas de envio de e-mails de marketing e newsletters. 45,36\% responderam que utilizam serviços dessa natureza.

- Armazenamento. 45,36\% responderam que utilizam softwares de CN para armazenar dados;

- Sistemas de edição de texto, planilhas e apresentação. $44,33 \%$ dos respondentes utilizam esse tipo de software.

Observa-se que, dos serviços mais utilizados, mais de $60 \%$ das empresas têm serviços de e-mail na nuvem. A justificativa pode estar nos seguintes itens:

- Serviços de e-mail sob a abordagem de CN geralmente oferecem um SLA de 99,9\%. Esse nível de disponibilidade dificilmente seria superado por aplicações sob a abordagem tradicional de TI com um custo razoável;

- Em termos de custo, é mais vantajoso para a organização contratar um fornecedor de serviço de e-mail sob a abordagem de $\mathrm{CN}$ porque com isso ela não terá que arcar com gastos de manutenção de servidores, custos de energia elétrica e funcionários de TI para administrar e configurar os serviços;

Quando à hospedagem de aplicações, o que se observa é uma migração de serviços sob a abordagem tradicional para a $\mathrm{CN}$. Além dos benefícios do modelo de $\mathrm{CN}$ já discutidos na Seção 2.3, pode-se inferir que flexibilidade de configuração e os recursos oferecidos por fornecedores de IaaS justifiquem a migração para o modelo de CN. Serviços oferecidos nesta categoria são muito semelhantes aos serviços tradicionais de hospedagem, o que facilita a migração e a adaptação de novos usuários ao modelo de $\mathrm{CN}$.

O grande número de ocorrências da categoria "oferecimento de serviços de envio de e-mail de marketing e newsletters" pode ser justificado pela facilidade na qual esses serviços podem ser contratados e configurados quando disponibilizados em CN. Economicamente não compensa para a empresa manter uma infraestrutura e adquirir e configurar softwares de envio de e-mails e newsletters, fazendo com que a mesma busque soluções na $\mathrm{CN}$. 
Quanto ao armazenamento, o que pode explicar a alta porcentagem de serviços dessa categoria é aumento vertiginoso de volume de dados nas organizações. Os serviços oferecidos sob a abordagem de $\mathrm{CN}$ possuem mecanismos de cobrança que têm como base horas de utilização, quantidade de dados armazenados e quantidade de dados trafegados. Novamente, o usuário optará pela solução de $\mathrm{CN}$, pois caso contrário, seria necessário efetuar altos investimentos para se ter os mesmos benefícios em uma infraestrutura de TI local.

Já os processadores de texto, planilhas e apresentações são soluções que, sob a abordagem de $\mathrm{CN}$ possibilitam o compartilhamento (no qual múltiplos usuários podem trabalhar em um mesmo documento) e o trabalho distribuído (documentos ficam disponíveis na Internet).

b) Nacionalidade do fornecedor e importância do serviço. A Tabela 3 apresenta os dados sobre a motivação para escolha do fornecedor e a importância do serviço para a organização. As porcentagens quando somadas são superiores a $100 \%$ porque o usuário pôde escolher mais de uma opção.

Das motivações para escolha do fornecedor houve destaque para a redução de custos, maior disponibilidade, prestação de serviços e reputação do fornecedor. Essas quatro categorias apareceram como mais frequentes tanto para fornecedores externos quanto para fornecedores internos, embora não na mesma ordem.

Para os serviços cujos fornecedores são empresas nacionais, a redução de custos aparece como categoria mais frequente, seguida de maior disponibilidade. Esses resultados mostram que os usuários estão reconhecendo o apelo econômico do modelo de $\mathrm{CN}$ e compreendendo que serviços sob a abordagem de $\mathrm{CN}$ apresentam maior disponibilidade quando comparados a serviços tradicionais de TI, uma vez que tal característica é gerenciada por meio de SLAs rigorosos.

Com base nos dados, observa-se que os serviços oferecidos por empresas estrangeiras são considerados de alto impacto estratégico em $12 \%$ dos casos. Contudo, essa porcentagem cai para zero quando o fornecedor do serviço é nacional. Quanto ao impacto operacional observou-se novamente um maior número de ocorrências de casos de alto impacto na utilização de serviços de fornecedores estrangeiros $(20,6 \%$ para fornecedores estrangeiros e $12 \%$ para fornecedores nacionais). No geral, tanto para fornecedores nacionais quanto para estrangeiros há uma maior concentração de serviços com baixo ou médio impacto operacional e estratégico. Este fato mostra que, embora haja uma confiança no modelo de $\mathrm{CN}$, os usuários iniciaram o processo de adoção pela utilização de serviços de baixo impacto para a organização.

Tabela 3. Motivação para escolha do fornecedor e impactos do serviço

\begin{tabular}{|c|c|c|c|c|c|c|}
\hline & Motivação da Escolha & $\%$ & $\begin{array}{c}\text { Impacto } \\
\text { Estratégico }\end{array}$ & $\%$ & $\begin{array}{c}\text { Impacto } \\
\text { Operacional }\end{array}$ & $\%$ \\
\hline & Redução de custo & 64,0 & \multirow{4}{*}{ Baixo } & \multirow{4}{*}{48,0} & \multirow{4}{*}{ Baixo } & \multirow{4}{*}{48,0} \\
\hline & Maior disponibilidade & 52,0 & & & & \\
\hline 正 & Prestação de serviço & 44,0 & & & & \\
\hline ة & Reputação Fornecedor & 32,0 & & & & \\
\hline 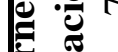 & Segurança da informação & 28,0 & \multirow{4}{*}{ Médio } & \multirow{4}{*}{52,0} & \multirow{4}{*}{ Médio } & \multirow{4}{*}{40,0} \\
\hline & Acesso conhecimento & 16,0 & & & & \\
\hline & Gestão de recursos humanos & 12,0 & & & & \\
\hline & Atividades rotineiras & 8,0 & & & & \\
\hline
\end{tabular}




\begin{tabular}{|c|c|c|c|c|c|c|}
\hline & Flutuação carga de trabalho & 8,0 & \multirow{4}{*}{ Alto } & \multirow{4}{*}{0,0} & \multirow{4}{*}{ Alto } & \multirow{4}{*}{12,0} \\
\hline & Atividade alto grau particularidade & 4,0 & & & & \\
\hline & Imposições externas & 4,0 & & & & \\
\hline & Escolha Fornecedor - Outros & 4,0 & & & & \\
\hline & & & Total & 100,0 & Total & 100,0 \\
\hline \multirow{12}{*}{ 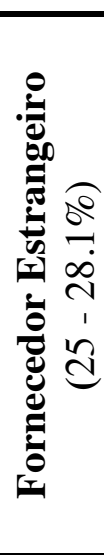 } & Reputação Fornecedor & 71,9 & \multirow{4}{*}{ Baixo } & \multirow{4}{*}{29,7} & \multirow{4}{*}{ Baixo } & \multirow{4}{*}{38,1} \\
\hline & Maior disponibilidade & 67,2 & & & & \\
\hline & Redução de custo & 59,4 & & & & \\
\hline & Prestação de serviço & 45,3 & & & & \\
\hline & Segurança da informação & 45,3 & \multirow{4}{*}{ Médio } & \multirow{4}{*}{56,3} & \multirow{4}{*}{ Médio } & \multirow{4}{*}{41,3} \\
\hline & Acesso conhecimento & 29,7 & & & & \\
\hline & Atividades rotineiras & 21,9 & & & & \\
\hline & Flutuação carga de trabalho & 15,6 & & & & \\
\hline & Atividade alto grau particularidade & 9,4 & \multirow{4}{*}{ Alto } & \multirow{4}{*}{14,1} & \multirow{4}{*}{ Alto } & \multirow{4}{*}{20,6} \\
\hline & Gestão de recursos humanos & 3,1 & & & & \\
\hline & Imposições externas & 3,1 & & & & \\
\hline & Escolha Fornecedor - Outros & 3,1 & & & & \\
\hline & & & Total & 100,0 & Total & 100,0 \\
\hline
\end{tabular}

c) Forma de disponibilização, localização física dos dados, pagamento e disponibilidade. A Tabela 4 apresenta os dados sobre a forma de disponibilização dos serviços de $\mathrm{CN}$, a disponibilidade, a localização e a forma de pagamento. Para cada coluna foi considerado um número de casos diferente, já que o número de valores faltantes para cada dimensão pode mudar. Contudo, as porcentagens totais de cada cruzamento são apresentadas nas linhas intermediárias entre as faixas da forma de disponibilização. Assim, os somatórios dos totais só são válidos para as colunas. A disponibilidade foi considerada boa ou ótima tanto para nuvens públicas quando para nuvens privadas e híbridas. Para a maioria dos casos não é possível escolher a localização física exata dos dados e os planos mensais e anuais são as formas predominantes de pagamento.

Tabela 4. Disponibilidade, localização dos dados e forma de pagamento

\begin{tabular}{|c|c|c|c|c|c|c|}
\hline \multirow{2}{*}{$\begin{array}{c}\text { Forma de } \\
\text { Disponibilização }\end{array}$} & \multicolumn{2}{|c|}{ Disponibilidade } & \multicolumn{2}{|c|}{ Localização física dos dados } & \multicolumn{2}{|c|}{ Forma de Pagamento } \\
\hline & Nível & $\%$ & Nível & $\%$ & Nível & $\%$ \\
\hline \multirow{5}{*}{ Pública } & Péssima & 0,0 & Não escolhe & 31,9 & Por hora & 6,5 \\
\hline & Ruim & 1,1 & País & 11,7 & Planos mensais/anuais & 21,7 \\
\hline & Regular & 1,1 & Estado & 0,0 & Usuários ativos & 4,3 \\
\hline & Boa & 16,5 & Cidade & 2,1 & Gratuito & 15,2 \\
\hline & Ótima & 29,7 & Datacenter & 2,1 & Outros & 0,0 \\
\hline Total & & 48,4 & & 47,9 & & 47,8 \\
\hline \multirow{5}{*}{ Privada } & Péssima & 0,0 & Não escolhe & 12,8 & Por hora & 2,2 \\
\hline & Ruim & 1,1 & País & 10,6 & Planos mensais/anuais & 19,6 \\
\hline & Regular & 1,1 & Estado & 0,0 & Usuários ativos & 4,3 \\
\hline & Boa & 15,4 & Cidade & 1,1 & Gratuito & 4,3 \\
\hline & Ótima & 12,1 & Datacenter & 5,3 & Outros & 0,0 \\
\hline Total & & 29,7 & & 29,8 & & 30,4 \\
\hline Híbrida & Péssima & 0,0 & Não escolhe & 10,6 & Por hora & 2,2 \\
\hline
\end{tabular}




\begin{tabular}{|c|c|r|c|r|c|r|}
\hline \multirow{7}{*}{ Total } & Ruim & 0,0 & País & 8,5 & Planos mensais/anuais & 12,0 \\
\cline { 2 - 7 } & Regular & 0,0 & Estado & 0,0 & Usuários ativos & 2,2 \\
\cline { 2 - 7 } & Boa & 5 & Cidade & 0,0 & Gratuito & 4,3 \\
\cline { 2 - 7 } & Ótima & 16,5 & Datacenter & 3,2 & Outros & 1,1 \\
\hline & \multicolumn{3}{|c}{22,0} & \multicolumn{3}{c}{21,7} \\
\hline
\end{tabular}

\section{Conclusões}

Este trabalho teve como objetivo analisar a adoção da CN por organizações brasileiras. Foi utilizada uma amostra não probabilística contendo 96 casos e os dados foram coletados por meio de um questionário enviado por e-mail e divulgado em fóruns de TI entre Julho de Agosto de 2012. Durante o processo de coleta dos dados, houve dificuldade em se encontrar empresas que utilizassem a $\mathrm{CN}$. $\mathrm{O}$ questionário foi amplamente divulgado em listas de e-mails, grupos e fóruns de TI para que se pudesse ter um número razoável de respondentes. Para a análise dos dados foram utilizadas técnicas não paramétricas e análise de agrupamentos.

A escolha de uma amostra não probabilística faz com que não seja possível generalizar os resultados. Além disso, as técnicas usadas para a análise dos dados não permitem descobrir as razões que conduzem ao fenômeno, apenas as relações entre as variáveis. Contudo, como o estudo é exploratório, os resultados podem servir como base para futuros trabalhos de natureza explicativa. Os resultados e contribuições estão apresentados de acordo com os objetivos propostos.

\subsection{Serviços de CN Adotados pelas Organizações Brasileiras}

A pesquisa mostrou predominância de serviços de $\mathrm{CN}$ genéricos, como e-mail, hospedagem, armazenamento e aplicativos de processamento de textos, planilhas e apresentações. Este fato mostra uma tendência do mercado de uso da TI como uma utilidade, ou seja, o que não é específico é terceirizado sob forma de $\mathrm{CN}$.

Os dados mostraram que apenas $25,77 \%$ dos serviços oferecidos no Brasil são fornecidos por empresas nacionais. A predominância de serviços de empresas estrangeiras pode ser explicada por diversos fatores, dentre eles:

a) Pioneirismo - os primeiros serviços de $\mathrm{CN}$ a se popularizarem foram os serviços oferecidos pelas empresas Amazon, Microsoft e Google. Os dados mostraram que as três empresas acima citadas são responsáveis pelo fornecimento de mais da metade dos serviços de $\mathrm{CN}$ utilizados por organizações brasileiras.

b) Pagamento - nos serviços de $\mathrm{CN}$ oferecidos por empresas estrangeiras a forma de pagamento predominante é o pagamento por utilização. $\mathrm{O}$ usuário paga por hora de uso e a adição ou remoção de recursos é feita rapidamente e sem interações com o fornecedor. Já os fornecedores nacionais adotam os planos mensais ou anuais como forma de pagamento pelos serviços.

Os critérios mais relevantes levados em consideração para escolha do fornecedor do serviço de $\mathrm{CN}$ foram a redução de custos e a maior disponibilidade do serviço. A redução de custos está diretamente ligada à característica da $\mathrm{CN}$ de pagamento por utilização. $\mathrm{O}$ usuário não precisa adquirir equipamentos de TI e, dessa forma, também não há custos de manutenção, recursos humanos, energia elétrica, refrigeração, dentre outros. 
Quanto à disponibilidade dos serviços, deve-se considerar que para a maioria dos fornecedores de serviços de CN o SLA é de 99,5\%, chegando em alguns casos a 99,95\% (Amazon EC2).

Quando à forma de pagamento pelos serviços houve predominância do pagamento com base em planos anuais ou mensais, seguido por pagamento por hora e por usuários ativos. As análises dos dados mostraram que para fornecedores nacionais predominam os planos mensais e anuais como forma de pagamento. Já os fornecedores estrangeiros adotam pagamento por utilização ou gratuidade (até certo limite).

Apesar de genéricos, os serviços de $\mathrm{CN}$ forma considerados importantes tanto do ponto de vista operacional quanto estratégico. Os dados mostraram que para $37,11 \%$ dos serviços de $\mathrm{CN}$ pesquisados uma hora de interrupção causa prejuízos aos clientes. No âmbito estratégico, os custos e o aumento da produtividade foram os itens mais citados.

$\mathrm{Na}$ característica tempo de alocação de recursos predominaram os serviços com tempo inferior a uma hora e entre uma e três horas. Ou seja, no geral, para os serviços de $\mathrm{CN}$ disponíveis no mercado, a configuração de novos recursos é relativamente rápida quando comparada ao fornecimento tradicional de TI. Já para o número de interações com o fornecedor na configuração de novos recursos, quase $70 \%$ dos serviços não é necessária nenhuma interação com o fornecedor para alterar parâmetros do serviço contratado. A disponibilidade dos serviços de $\mathrm{CN}$ foi classificada como ótima por quase $60 \%$ dos respondentes, mostrando que os serviços de $\mathrm{CN}$ consumidos por organizações brasileiras atingiram um nível de maturidade compatível com a definição de $\mathrm{CN}$ utilizada como base para estre trabalho.

Quanto à localização dos dados, para a maioria dos serviços pesquisados o usuário não tem a opção de escolher a localização física exata de onde os dados serão armazenados. Para a realidade brasileira isso pode significar problemas tanto no âmbito da latência (caso os servidores estejam em outro país) quanto da legislação (governos externos podem ter acesso aos dados por questões jurídicas).

Já a forma de disponibilização mais frequente nos serviços pesquisados foi a pública, ou seja, os serviços podem ser contratados por qualquer usuário interessado

\subsection{Sugestões de Pesquisa}

Recomenda-se a elaboração de novas pesquisas com base neste trabalho. Dentre as inúmeras alternativas, pode-se citar:

- Replicar o mesmo modelo de pesquisa considerando outras características do serviço de CN;

- Analisar a adoção da CN no Brasil do ponto de vista do fornecedor, ou seja, avaliar as possíveis dificuldades técnicas e gerenciais no desenvolvimento de produtos com maior nível de $\mathrm{CN}$;

- Desenvolver novas pesquisas a partir de amostras probabilísticas que permitam a generalização dos resultados e, portanto, o aumento do conhecimento sobre o fenômeno da CN no Brasil; 


\section{Referências}

AMAZON. Amazon Elastic Compute Cloud (Amazon EC2). Disponível em: $<$ http://aws.amazon.com/pt/ec2/>. Acesso em: 29/1/2012.

APPLEGATE, L. M.; AUSTIN, R. D.; MCFARLAN, F. W. Corporate Information Strategy and Management. $6^{\mathrm{a}}$ ed. McGraw-Hill, 2003.

ARMBRUST, M.; FOX, A.; GRIFFITH, R.; JOSEPH, A.; KATZ, R. Above the clouds: A Berkeley view of cloud computing. Technical Report n. UCBEECS - 2009-28. CA (US): University of California, Berkeley - Electrical Engineering and Computer Sciences, 2009. Disponível em: < http://www.eecs.berkeley.edu/Pubs/TechRpts/2009/EECS-2009-28.pdf >. Acesso em: 23-10-2012.

BABBIE, E. The Practice of Social Research. 12th edition. Belmont, CA: Wadsworth, 2010 .

ISACA. Computação em Nuvem: Benefícios para o Negócio com Perspectivas de Segurança, Governança e Qualidade. Documento Técnico da ISACA sobre Tecnologias Emergentes, 2009. Disponível em: <http://www.isaca.org/KnowledgeCenter/Research/Documents/Cloud_WP_Portuguese_23Feb2011.pdf?id=7b50eb1c093f-4835-b8c5-c8c9a7ab71d3>. Acesso em 28/10/2012.

JEFFERY, K.; NEIDECKER-LUTZ, B. The Future of Cloud Computing Opportunities for European Cloud Computing Beyond 2010. Expert Group Report, Information Society and Media. European Comission, 2010. Disponível em $<$ http://cordis.europa.eu/fp7/ict/ssai/docs/cloud-report-final.pdf $>$. Acesso em: 28/10/2012.

MELL, P.; GRANCE, T. The NIST Definition of Cloud Computing. NIST Special Publication. Gaithersburg, MD (US): National Institute of Standards and Technology, v. 800, p. 145, 2011. Disponível em: $<$ http://docs.ismgcorp.com/files/external/Draft-SP-800-145_cloud-definition.pdf>. Acesso em: 4/1/2012.

MOTAHARI-NEZHAD, H. R.; STEPHENSON, B.; SINGHAL, S. Outsourcing Business to Cloud Computing Services: Opportunities and Challenges. HP Laboratories Development. Technical Report HPL-2009-23 ,2009. Disponível em $<$ http://www.hpl.hp.com/techreports/2009/HPL-2009-23.pdf >. Acesso em 28/10/2012.

NEUMAN, W. Basics of Social Research: Qualitative and Quantitative Approaches. 2nd ed. Boston, MA (US): Pearson Education, 2007.

SCHAFFER, H. E. X as a Service, Cloud Computing, and the Need for Good Judgment. IT Professional, v. 11, n. 5, p. 4-5, 2009. IEEE. Disponível em: <http://www.ncbi.nlm.nih.gov/pubmed/21868581>. Acesso em: 28/2/2012.

VAQUERO, L. M.; RODERO-MERINO, L.; CACERES, J.; LINDNER, M. A Break in the Clouds: Towards a Cloud Definition. In: SIGCOMM Computer Communication Review. New York, NY, (US): ACM, v. 39, n. 1, p. 50-55, 2009.

WANG, L.; TAO, J.; KUNZE, M. et al. Scientific Cloud Computing: Early Definition and Experience. In: 10th IEEE International Conference on High Performance 
Computing and Communications. Dalian, China: Institute of Electrical and Electronics Engineers (IEEE), p. 825-830, 2008.

YIN, R. K. Estudo de Caso: Planejamento e Métodos. 2a ed. São Paulo, SP: Bookman, 2001. 